\title{
Nanomedical Theranostics in Cardiovascular Disease
}

\author{
Jun Tang • Mark E. Lobatto • Joanna C. Read • \\ Aneta J. Mieszawska $\cdot$ Zahi A. Fayad • \\ Willem J. M. Mulder
}

Published online: 29 November 2011

(C) The Author(s) 2011. This article is published with open access at Springerlink.com

\begin{abstract}
Cardiovascular disease (CVD) is the leading cause of morbidity and mortality worldwide. New diagnostic and therapeutic strategies are needed to mitigate this public health issue. Advances in nanotechnology have generated innovative strategies for diagnosis and therapy in a variety of diseases, foremost in cancer. Based on these studies, a novel concept referred to as nanomedical theranostics, or the combinatory application of nanoparticulate agents to allow diagnostic therapy, is being explored to enable image-guided, personalized, or targeted treatment. Preclinically, theranostics have been gradually applied to CVD with several interesting and encouraging findings. This article summarizes studies and challenges of nanotheranostic strategies in CVD. It also evaluates nanotheranostic strategies that may potentially be utilized to benefit patients.
\end{abstract}

Keywords Cardiovascular disease - Nanoparticle . Theranostics · Diagnostics · Therapeutics · Cardiac molecular imaging

\section{Introduction}

Cardiovascular disease (CVD) is the most prevalent disease in the United States, accounting for 1 out of 3 deaths in 2007, with an estimated cost of 286 billion US dollars [1 ${ }^{\bullet}$.

J. Tang • M. E. Lobatto · J. C. Read - A. J. Mieszawska •

Z. A. Fayad - W. J. M. Mulder $(\bowtie)$

Translational and Molecular Imaging Institute,

Mount Sinai School of Medicine,

One Gustave L. Levy Place, Box 1234, New York, NY 10029, USA

e-mail: willem.mulder@mountsinai.org

M. E. Lobatto

Department of Vascular Medicine, Academic Medical Center,

Amsterdam, The Netherlands
New diagnostic and therapeutic strategies are needed as patients present themselves at late stages of disease or, more commonly, after cardiovascular events. In addition, subsets of patients do not respond to current therapeutic methods [2].

An improved understanding of atherosclerosis, the underlying cause of the majority of CVDs, has identified several biological processes that aggravate this syndrome. In the early stages of atherosclerosis, the endothelium, a continuous layer of endothelial cells (ECs) lining the vessel wall, starts - under influence of cardiovascular disease risk factors such as hypertension, abnormal blood lipid levels or smoking - to express high levels of adhesion molecules and have increased cellular gaps. This initiates accumulation of low-density lipoproteins (LDL) as well as activated macrophages in the subendothelial layer. In later stages, high levels of extracellular proteinases, apoptotic cells, and accumulating radicals contribute to the progression of atherosclerotic plaques. As the plaque progresses, hypoxia-driven angiogenesis triggers the formation of new blood vessels. In the final stage of atherosclerosis, tissue factors from plaques are exposed to blood and induce thrombosis, resulting in clinical symptoms such as myocardial infarction or stroke [3, 4•].

In biomedicine, nanoparticles, typically defined as materials with at least one dimension of $100 \mathrm{~nm}$ or less, have been exploited for both diagnostic and therapeutic purposes [5]. Certain contrast agents and potent drugs may exhibit a poor blood half-life and high systemic toxicity, which can potentially be improved by their encapsulation within nanoparticles. In addition, nanoparticles can increase the effectiveness of many diagnostic or therapeutic agents. Decorating nanoparticles with targeting molecules against biomarkers of a disease facilitates their specific delivery and accumulation within pathological tissue [6]. 
Although the application of nanoparticles to diagnose and treat atherosclerosis is a relatively young field, a number of studies have shown its growing potential. In atherosclerotic plaques, the high expression levels of certain molecules, such as $\alpha_{\mathrm{v}} \beta_{3}$-integrin on ECs, allow these cells to be directly reached from the circulation and therefore to be specifically targeted by nanoparticles [7]. In addition, long-circulating nanoparticles can penetrate plaques through the enlarged cellular gaps within the endothelial lining and thereby reach intra-plaque components. Nanoparticles can also be functionalized to target molecules or cells within the plaque, such as oxidized LDL or macrophages. The ability to target these processes allows targeted therapy of atherosclerosis with nanoparticles [8], while nanoparticles loaded with contrast agents can serve as imaging reporters [9]. For example, reconstituted highdensity lipoproteins can be incorporated with contrastgenerating materials to enable target-specific imaging of macrophages in plaques using a variety of imaging modalities, including MRI and CT [10].

Nanoparticles that hold both therapeutic drugs and diagnostic labels are popularly referred to as theranostic nanoparticles. Nevertheless, the field on nanotheranostics is not solely focused on the use of theranostic nanoparticles, but also includes a variety of combinations of nanomedicine, (targeted) therapy, and imaging [11], with the ultimate aim to improve diagnosis, achieve targeted therapy, or monitor response to therapy. Due to their complexity, it is unlikely that theranostic nanoparticles will be consistently applied clinically. However, nanotheranostic approaches may have significant benefits. Moreover, compared to dedicated therapeutic nanoparticles, theranostic nanoparticles provide interesting additional benefits that can facilitate and speed up preclinical development. Advantages include the ability to monitor biodistribution and investigate nanoparticle dynamics in the body over time [12]. In addition, ex vivo imaging techniques, such as transmission electron microscopy (TEM) and fluorescence microscopy, are frequently used for co-localization purposes and to corroborate in vivo findings. Altogether, nanotheranostics has the potential to accelerate and improve the development of novel therapeutic strategies for CVD.

\section{Overview of Studies}

Nanotheranostic studies can roughly be classified into three distinct categories. First, the use of nanoparticulate contrast agent-aided imaging to evaluate the efficacy of therapy; second, the use of imaging to evaluate nanotherapy; third, the use of theranostic nanoparticles for the purpose of diagnosing, treating, and subsequently evaluating disease (Fig. 1).

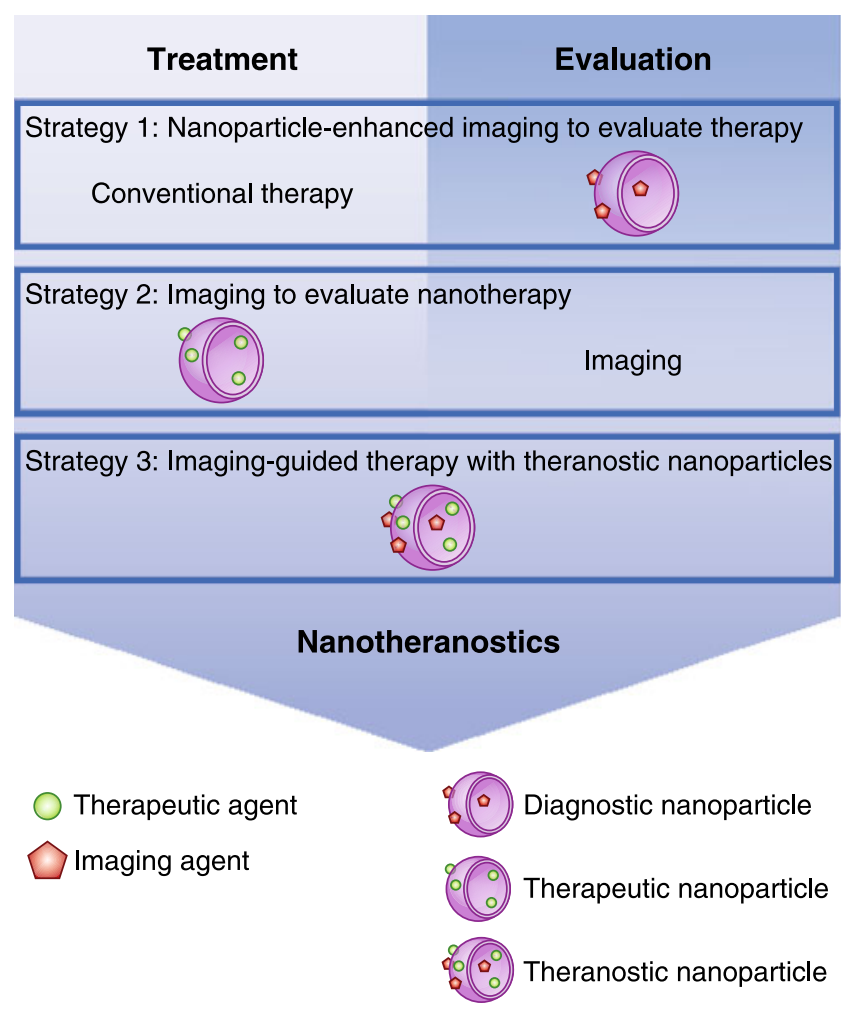

Fig. 1 Three strategies used in nanotheranostic studies for cardiovascular disease

Nanoparticle-Aided Imaging to Evaluate Efficacy of Therapy

\section{Atherosclerosis}

It is well established that ultrasmall particles of iron oxide (USPIO) are taken up by macrophages in high-risk atherosclerotic plaques in both animals and humans [13, 14]. Following up on this, Morris et al. [15] used USPIOenhanced MRI to evaluate therapeutic effects of a p38 kinase inhibitor, a critical molecule in the immune response, in an apolipoprotein E-deficient (ApoE KO) mouse model. The MRI studies revealed decreased accumulation of USPIO in the aortic root and arch area. In addition, ex vivo histology confirmed that the majority of USPIO were associated with macrophages. Their findings indicate the potential of USPIO-enhanced MRI as a method to evaluate therapeutic effects noninvasively [15]. In patients, this method has been used to evaluate statin treatment and the effects of high-dose and a low-dose of atorvastatin on carotid plaques. Continuation of a high-dose treatment for 12 weeks decreased USPIO uptake in carotid plaques, while the low-dose treatment had no significant effect. This study represents one of the first clinical examples of nanoparticle-aided noninvasive imaging for the evaluation of drug therapy in patients with atherosclerosis [16•]. 
Peripheral artery disease (PAD) refers to luminal narrowing of peripheral arteries, which subsequently leads to reduced blood flow and oxygenation of tissue, resulting in PAD symptoms such as claudication or critical limb ischemia [17]. L-arginine can increase arteriogenesis, a biological process that has the potential to decrease PAD symptoms by increasing blood flow and oxygenation. Winter et al. [18] applied a nanoparticle formulation, with a diameter $<300 \mathrm{~nm}$, specific to $\alpha_{\mathrm{v}} \beta_{3}$-integrin to evaluate if targeted nanoparticles can detect the neovascular response to arteriogenic treatment in a rabbit model. Ischemic hind limbs showed elevated signal enhancement in response to treatment with in vivo nanoparticle-enhanced MRI (Fig. 2a), which was corroborated with ex vivo X-ray angiography. This method might lead to an earlier and more accurate detection of collateral vessel growth in response to treatment in PAD patients [18].

\section{Evaluation of Nanotherapy by Imaging}

\section{Vascular Injury and Stenosis Inhibition}

The luminal narrowing of blood vessels due to atherosclerosis progression is referred to as stenosis. Treating stenotic vessels with balloon dilatation and stent placement are wellaccepted strategies for revascularization. However, restenosis in dilated and stented vessels may elicit other complications, such as myocardial damage and impeded peripheral circulation. Drug-eluting stents can reduce instent restenosis but can also interfere with endothelial healing, which can cause late-stent thrombosis [19]. Cyrus et al. [20] developed rapamycin-loaded $\alpha_{\mathrm{v}} \beta_{3}$-integrintargeted paramagnetic nanoparticles with the purpose of reducing stenosis after balloon injury without inhibiting endothelial healing. The nanoparticles, with a diameter of $193 \mathrm{~nm}$, were intramurally injected after balloon injury of the femoral artery of a rabbit model. MR angiograms were able to track nanoparticle uptake in injured femoral arteries in vivo, also showing patent and less stenotic arteries after 2 weeks. These findings were confirmed by histology with significantly reduced stenosis, while endothelial healing remained unaffected. This platform could be a potential alternative to drug-eluting stents or serve as a supplement to current therapy [20]. Using a different form of focal therapy, Chorny et al. [21] developed novel paclitaxelloaded magnetic nanoparticles (MNPs) that have a diameter of $263 \mathrm{~nm}$ and exploit magnetic properties of stents. By applying a uniform magnetic field after the administration of MNPs, they specifically accumulate in stented areas, achieving a high local concentration of paclitaxel. Fluorescence imaging and histology were used to evaluate therapeutic effects, finding local accumulation of MNPs that delayed cell proliferation and in-stent restenosis. The advantages of this system include an improved therapeutic effect over nanoparticles without the applied magnetic field and the possibility to adjust local drug concentrations by controlling the external magnetic field [21].

\section{Peripheral Arterial Disease: Improving Perfusion}

Cytokines, such as vascular endothelial growth factor (VEGF), are potent therapeutic compounds. However, they exhibit limited circulatory half-life and poor specificity. In a study by Kim et al. [22], a targeted gold nanoparticle was developed with a size of $80 \mathrm{~nm}$ that specifically delivers VEGF to ischemic tissue for the purpose of increasing therapeutic angiogenesis. Laser Doppler perfusion imaging was used to noninvasively evaluate therapeutic effects. In this study, VEGF-conjugated gold nanoparticles increased perfusion of ischemic musculature by 1.7 -fold compared to free VEGF in a murine hind limb model (Fig. 2b) [22]. This study exemplifies the ability of nanoparticles to increase the efficacy of certain molecules by increasing their circulatory half-lives and specificity, while also reducing undesired side effects.

\section{Limiting Reperfusion Injury in Acute Myocardial Infarction}

Adenosine is being investigated as an adjunct to reperfusion therapy for acute myocardial infarction as it shows cardioprotective effects in clinical trials [23]. Disadvantages of adenosine include an extremely short half-life (1 to $2 \mathrm{~s}$ ) and the side effects such as hypotension and bradycardia. Takahama et al. [24] injected a liposomal formulation of adenosine, with a mean diameter of $134 \mathrm{~nm}$, intravenously in an ischemic/reperfusion myocardial rat model prior to the onset of reperfusion. TEM and fluorescence images showed the accumulation of liposomes in infarcted areas. Liposomal adenosine showed an enhanced circulation time, reduced side effects, and significantly reduced myocardial infarction size compared to free adenosine controls [24].

\section{Thrombosis Treatments}

Phe[D]-Pro-Arg-Chloromethylketone (PPACK) has a very specific affinity to thrombin, a key molecule in the coagulation cascade, and has the potential to be applied as an anti-thrombotic agent. In a study by Myerson et al. [25], PPACK molecules were conjugated to perfluorocarbon nanoparticles, with a final size of $160.5 \mathrm{~nm}$. In a photochemical injury thrombosis mouse model, they showed that the antithrombosis efficacy of PPACK nanoparticles was higher than free PPACK and heparin, as PPACK nanoparticle-treated mice developed thrombosis at later time points. Ex vivo proton MRI and ${ }^{19} \mathrm{~F}$ MRI showed that the nanoparticles specifically accumulated at thrombotic sites [25]. In another 
A Molecular Imaging of Angiogenesis

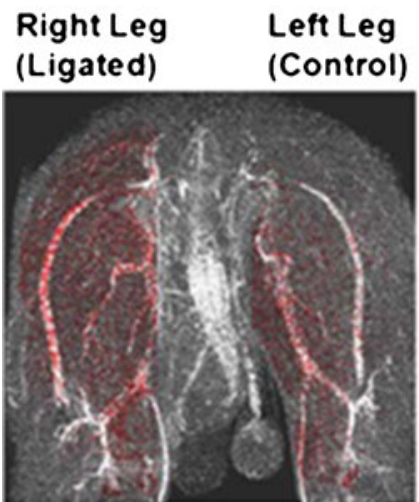

Tap Water

$\begin{array}{ll}\text { Right Leg } & \text { Left Leg } \\ \text { (Ligated) } & \text { (Control) }\end{array}$

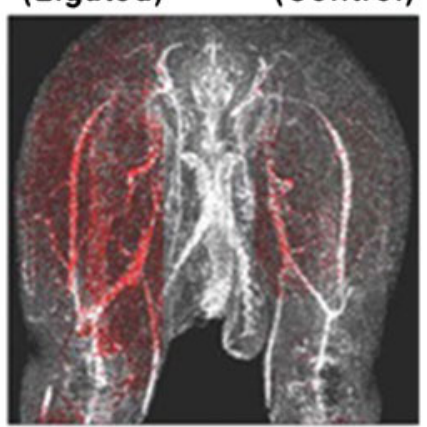

L-Arginine
C
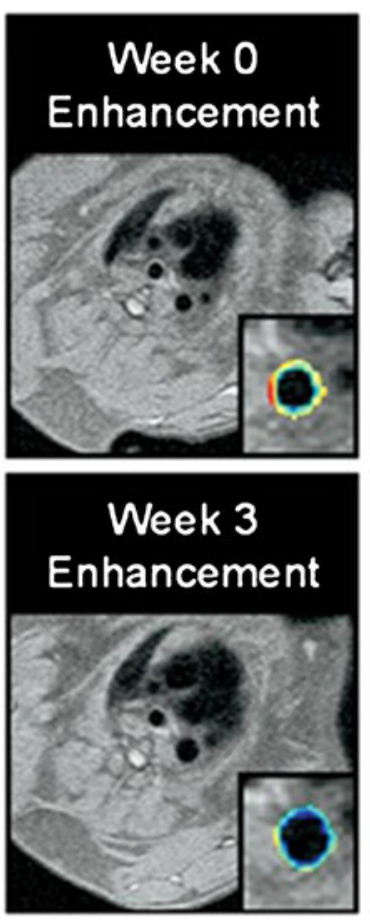

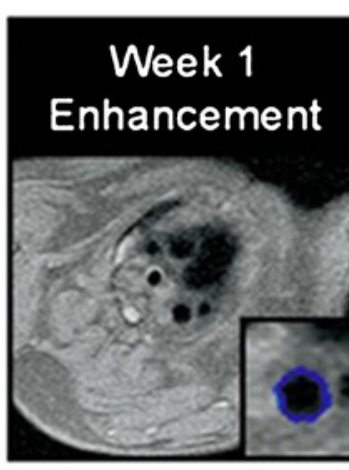

Week 4 Enhancement

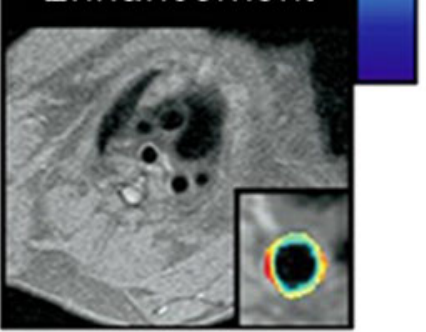

B
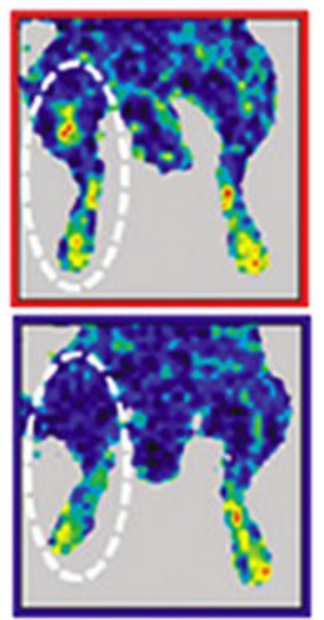

D
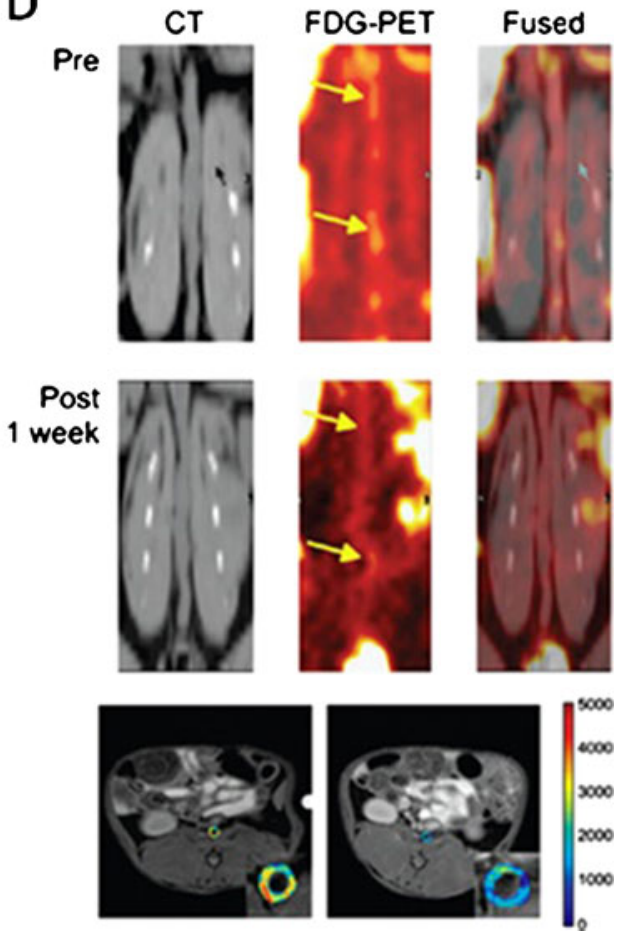

Fig. 2 Studies that used different nanotheranostic strategies. a, Larginine treatment increases arteriogenesis in ischemic tissue. Right femoral arteries of rabbits were surgically ligated, while the left femoral arteries served as controls. After ligation rabbits received 10 days' treatment of L-arginine or tap water and were imaged by $\alpha_{v} \beta_{3}$-integrin-targeted nanoparticle-aided $T_{1}$-weighted MRI. Red color-coded signal enhancement indicates that arteriogenesis is more prevalent after L-arginine treatment. (Adapted from Winter et al. [18]; with permission.) $\mathbf{b}$, Comparison of perfusion after administration of VEGF-conjugated gold nanoparticles in ischemic hind limbs. After ligation of the femoral artery either VEGF-conjugated nanoparticles or free VEGF was administered. Five weeks post-treatment, VEGFconjugated nanoparticle-treated mice have improved blood perfusion (upper panel) compared to free VEGF-treated mice (lower panel) shown by laser Doppler perfusion imaging. (Adapted from Kim et al. [22]; with permission.) c, A single dose of $\alpha_{v} \beta_{3}$-integrin-targeted fumagillin-loaded nanoparticle treatment suppresses angiogenesis in aortic wall. Cardiac magnetic imaging was performed on hyperlipidemic rabbits at the moment of nanoparticle treatment (week 0), and 1,3 , or 4 weeks post-treatment. The red overlays on the arterial wall are color-coded percentages of signal enhancement. (Adapted from Winter et al. [30]; with permission.) d, Liposome-encapsulated glucocorticoids have anti-inflammatory effects on atherosclerotic plaques of rabbits. Rabbits received a single dose of liposomes and were imaged by ${ }^{18} \mathrm{~F}-\mathrm{FDG}-\mathrm{PET} / \mathrm{CT}$ and DCE-MRI pre and post treatment. The top two panels show coronal CT, ${ }^{18} \mathrm{~F}-\mathrm{FDG}-\mathrm{PET}$, and fused imaging slices of the abdominal aorta pre and 1 week post treatment. Yellow arrows indicate patchy areas with decreased FDG uptake after treatment. DCE-MRI shows a decrease in area under the curve (AUC) 2 days post treatment (right panel) compared to baseline (left panel). The color overlays on the MRI images are expressed in percentage of AUC, which has shown to correlate with neovascularization in atherosclerotic arterial walls. (Adapted from Lobatto et al. [31•]; with permission) 
study, Peters et al. [26] developed a micelle-based theranostic nanoparticle that can be loaded with anti-coagulation drugs and peptides that specifically bind to clotted plasma proteins. The 17-nm nanoparticles showed specific in vivo targeting to atherosclerotic plaques as shown by ex vivo fluorescence imaging. When loaded with hirulog, an anti-thrombosis peptide, the nanoparticles showed better anti-thrombosis effects than free hirulog at the same molar concentrations in ApoE KO mice [26].

Theranostic Nanoparticles for Simultaneous Treatment and Evaluation of Disease

\section{Inhibiting Restenosis}

As previously mentioned, restenosis frequently occurs after revascularization treatment. Smooth muscle cell (SMC) proliferation in the vessel wall is believed to promote restenosis and, therefore, inhibition of this process has been investigated as a strategy to prevent restenosis [27]. In a study by Lanza et al. [28], perfluorocarbon nanoparticles were used to deliver doxorubicin and paclitaxel, two potent anti-proliferation drugs, to SMCs in the arterial wall of restenotic vessels. The nanoparticles, with a size of $250 \mathrm{~nm}$, were targeted to tissue factor expressed by SMCs and their inhibited proliferation was observed in vitro. $\mathrm{T}_{1}$-weighted MRI detected the uptake of particles while ${ }^{19} \mathrm{~F}$ MRI spectroscopy allowed distinguishing the nanoparticles from other tissue. Although no in vivo data were shown, this study exemplifies an interesting theranostic nanoparticle system for CVD [28].

\section{Stabilizing High-Risk Atherosclerotic Plaques}

Inhibiting angiogenesis has been shown to halt the progression of atherosclerosis [7]. In a study by Winter et al. [29], $\alpha_{v} \beta_{3}$-integrin targeted paramagnetic nanoparticles were loaded with the anti-angiogenesis drug fumagillin. MRI was used to evaluate the response to treatment by measuring changes in signal enhancement in atherosclerotic plaques. Decreased signal enhancement was seen after treatment, which indicated reduced angiogenesis. The in vivo evaluation was confirmed by ex vivo staining of microvessels [29]. This is the first study to show the application of dual-purpose theranostic nanoparticles in atherosclerosis. In a subsequent study, the theranostic advantages were further exploited by a combinatory treatment of anti-angiogenic nanoparticles and oral statins, which revealed synergistic effects. Using cardiac magnetic resonance molecular imaging, the therapeutic response could be evaluated over time (Fig. 2c) [30].

Due to the inflammatory nature of atherosclerotic plaques, anti-inflammatory drugs such as glucocorticoids have been proposed as a treatment, though the systemic side effects impede its application in clinical medicine. Lobatto et al. [31•] used paramagnetically labeled liposomes, with a size of $103 \mathrm{~nm}$, as drug carriers to deliver glucocorticoids to atherosclerotic lesions in order to improve therapeutic efficacy. $\mathrm{T}_{1}$-weighted MRI showed that liposomes could be detected in atherosclerotic plaques. To evaluate therapeutic efficacy, ${ }^{18} \mathrm{~F}$-Fluoro-deoxy-glucose positron emission tomography $\left({ }^{18} \mathrm{~F}\right.$-FDG-PET), a noninvasive imaging modality that can detect plaque inflammation, showed reduced uptake of FDG in plaques. Furthermore, dynamic contrast-enhanced MRI (DCE-MRI), a method for detecting neovascularization, showed decreased uptake of contrast agents in the arterial wall (Fig. 2d) [31•].

McCarthy et al. [32] developed a novel theranostic platform to target macrophages. Dextran-coated iron oxide nanoparticles were loaded with near-infrared fluorophores and phototoxic agents and resulted in a particle size of $49.9 \mathrm{~nm}$. The nanoparticles specifically targeted macrophages and were activated by light to induce apoptosis in the targeted macrophages. In an ApoE KO mouse model the nanoparticles were shown to localize in atherosclerotic plaques by intravital fluorescence microscopy. Ex vivo histology staining showed that the nanoparticles induced massive death of macrophages, while they showed less skin toxicity than free phototoxic agents [32].

\section{Limitations and Conclusions}

Although various nanotheranostic strategies have been developed and significant advances have been achieved preclinically, some limitations in the present studies need to be addressed for future exploration and clinical translation. Currently, no standardized test procedures are available to evaluate the quality of nanoparticle therapeutics in general and their application for CVD specifically [33]. The same applies to the regulatory aspects. In addition, it is important to choose a proper disease model to test the efficacy of those nanoparticles that have shown potential in vitro. For the purpose of standardization, only well-established animal models should be used, such as the ApoE KO mouse model for atherosclerosis. Importantly, the acquired results should be critically interpreted, because of the differences in the pathology between animal models and humans. In mice, atherosclerotic plaques develop much faster and are smaller, which causes their buildup and function to be different. Mouse plaques are associated with limited neovascularization, and plaque rupture and thrombosis rarely occur. In addition, the arterial anatomy of mice is different from that of humans $[34,35]$. Therefore, nanoparticle studies that display promising results preclinically do not guarantee their suitability for clinical use. In certain 
theranostic nanoparticle formulations, toxic components are included, such as gadolinium, for diagnostic purposes [36]. This limits the use of such nanoparticles for long-term or high-frequency treatment in animal models and excludes their use in humans. In addition to the explored agents, novel diagnostic and therapeutic agents, including cleavable targeted peptides [37] and siRNA [38], are investigated in cardiovascular disease models and show great diagnostic and therapeutic potential. To enhance their function, these agents could be applied as nanoparticle formulations.

Far and foremost, it seems unlikely that theranostic nanoparticles will be applied to patients on a large scale. As mentioned before, therapeutic nanoparticles with imaging labels have several benefits in the developmental phase of nanotherapy, which may ultimately be stripped down to a pure therapeutic nanoparticle when moving forward to the clinic. On the other hand, the use of state-of-the-art atherosclerosis imaging techniques to evaluate novel therapies has recently been demonstrated in a multicenter clinical trial [39॰]. A similar setup to evaluate antiatherosclerotic nanotherapy would be a type of nanotheranostics that is likely to occur in the near future, while nanoparticle-enhanced imaging to evaluate traditional therapy has already been reported.

Acknowledgments Support for this work was partially provided by the International Atherosclerosis Society (M.E.L). This work was supported by the National Heart, Lung, and Blood Institute, National Institutes of Health, as a Program of Excellence in Nanotechnology (PEN) Award, Contract \#HHSN268201000045C, as well as by R01 EB009638 (Z.A.F.) and R01 CA155432 (W.J.M.M.).

Disclosure No potential conflicts of interest relevant to this article were reported.

Open Access This article is distributed under the terms of the Creative Commons Attribution Noncommercial License which permits any noncommercial use, distribution, and reproduction in any medium, provided the original author(s) and source are credited.

\section{References}

Papers of particular interest, published recently, have been highlighted as:

- Of importance

1. - Roger VL, Go AS, Lloyd-Jones DM, Adams RJ, Berry JD, Brown TM et al. Heart Disease and Stroke Statistics-2011 Update: A Report From the American Heart Association. Circulation.123(4):e18-e209. This article provides statistics of cardiovascular disease in the United States.

2. Libby P. The forgotten majority: unfinished business in cardiovascular risk reduction. J Am Coll Cardiol. 2005;46(7):1225-8.

3. Libby P, DiCarli M, Weissleder R. The vascular biology of atherosclerosis and imaging targets. J Nucl Med. 2010;51 Suppl $1: 33 \mathrm{~S}-7 \mathrm{~S}$.
4. - Hansson GK, Libby P. The immune response in atherosclerosis: a double-edged sword. Nat Rev Immunol. 2006;6(7):508-19. This article explains the pathophysiology of atherosclerosis.

5. Allen TM, Cullis PR. Drug delivery systems: entering the mainstream. Science. 2004;303(5665):1818-22.

6. Petros RA, DeSimone JM. Strategies in the design of nanoparticles for therapeutic applications. Nat Rev Drug Discov. 2010;9(8):615-27.

7. Moulton KS. Angiogenesis in atherosclerosis: gathering evidence beyond speculation. Curr Opin Lipidol. 2006;17(5):548-55.

8. Lanza GM, Winter PM, Caruthers SD, Hughes MS, Hu G, Schmieder AH, et al. Theragnostics for tumor and plaque angiogenesis with perfluorocarbon nanoemulsions. Angiogenesis. 2010;13(2):189-202.

9. Mulder WJ, Cormode DP, Hak S, Lobatto ME, Silvera S, Fayad ZA. Multimodality nanotracers for cardiovascular applications. Nat Clin Pract Cardiovasc Med. 2008;5 Suppl 2:S103-11.

10. Cormode DP, Skajaa T, van Schooneveld MM, Koole R, Jarzyna P, Lobatto ME, et al. Nanocrystal core high-density lipoproteins: a multimodality contrast agent platform. Nano Lett. 2008;8 (11):3715-23.

11. Janib SM, Moses AS, MacKay JA. Imaging and drug delivery using theranostic nanoparticles. Adv Drug Deliv Rev. 2010;62 (11):1052-63.

12. Sanhai WR, Sakamoto JH, Canady R, Ferrari M. Seven challenges for nanomedicine. Nat Nanotechnol. 2008;3(5):242-4.

13. Kooi ME, Cappendijk VC, Cleutjens KB, Kessels AG, Kitslaar PJ, Borgers $\mathrm{M}$, et al. Accumulation of ultrasmall superparamagnetic particles of iron oxide in human atherosclerotic plaques can be detected by in vivo magnetic resonance imaging. Circulation. 2003;107(19):2453-8.

14. Ruehm SG, Corot C, Vogt P, Kolb S, Debatin JF. Magnetic resonance imaging of atherosclerotic plaque with ultrasmall superparamagnetic particles of iron oxide in hyperlipidemic rabbits. Circulation. 2001;103(3):415-22.

15. Morris JB, Olzinski AR, Bernard RE, Aravindhan K, Mirabile $\mathrm{RC}$, Boyce R, et al. p38 MAPK inhibition reduces aortic ultrasmall superparamagnetic iron oxide uptake in a mouse model of atherosclerosis: MRI assessment. Arterioscler Thromb Vasc Biol. 2008;28(2):265-71.

16. • Tang TY, Howarth SP, Miller SR, Graves MJ, Patterson AJ, JM UK-I et al. The ATHEROMA (Atorvastatin Therapy: Effects on Reduction of Macrophage Activity) Study. Evaluation using ultrasmall superparamagnetic iron oxide-enhanced magnetic resonance imaging in carotid disease. J Am Coll Cardiol. 2009;53 (22):2039-50. This is the first clinical study that uses USPIOenhanced MRI to evaluate therapeutic effects of high-dose statin treatment.

17. Weinberg MD, Lau JF, Rosenfield K, Olin JW. Peripheral artery disease. Part 2: medical and endovascular treatment. Nat Rev Cardiol. 2011;8(8):429-41.

18. Winter PM, Caruthers SD, Allen JS, Cai K, Williams TA, Lanza $\mathrm{GM}$, et al. Molecular imaging of angiogenic therapy in peripheral vascular disease with alphanubeta3-integrin-targeted nanoparticles. Magn Reson Med. 2010;64(2):369-76.

19. O'Brien B, Carroll W. The evolution of cardiovascular stent materials and surfaces in response to clinical drivers: a review. Acta Biomater. 2009;5(4):945-58.

20. Cyrus T, Zhang H, Allen JS, Williams TA, Hu G, Caruthers SD, et al. Intramural delivery of rapamycin with alphavbeta3-targeted paramagnetic nanoparticles inhibits stenosis after balloon injury. Arterioscler Thromb Vasc Biol. 2008;28(5):820-6.

21. Chorny M, Fishbein I, Yellen BB, Alferiev IS, Bakay M, Ganta S, et al. Targeting stents with local delivery of paclitaxel-loaded magnetic nanoparticles using uniform fields. Proc Natl Acad Sci U S A. 2010;107(18):8346-51. 
22. Kim J, Cao L, Shvartsman D, Silva EA, Mooney DJ. Targeted delivery of nanoparticles to ischemic muscle for imaging and therapeutic angiogenesis. Nano Lett. 2011;11(2):694-700.

23. Ross AM, Gibbons RJ, Stone GW, Kloner RA, Alexander RW. A randomized, double-blinded, placebo-controlled multicenter trial of adenosine as an adjunct to reperfusion in the treatment of acute myocardial infarction (AMISTAD-II). J Am Coll Cardiol. 2005;45 (11): $1775-80$

24. Takahama H, Minamino T, Asanuma H, Fujita M, Asai T, Wakeno $\mathrm{M}$, et al. Prolonged targeting of ischemic/reperfused myocardium by liposomal adenosine augments cardioprotection in rats. J Am Coll Cardiol. 2009;53(8):709-17.

25. Myerson J, He L, Lanza G, Tollefsen D, Wickline S. Thrombininhibiting perfluorocarbon nanoparticles provide a novel strategy for the treatment and magnetic resonance imaging of acute thrombosis. J Thromb Haemost. 2011;9(7):1292-300.

26. Peters D, Kastantin M, Kotamraju VR, Karmali PP, Gujraty K, Tirrell $\mathrm{M}$, et al. Targeting atherosclerosis by using modular, multifunctional micelles. Proc Natl Acad Sci U S A. 2009;106 (24):9815-9.

27. Jukema JW, Verschuren JJ, Ahmed TA, Quax PH. Restenosis after PCI. Part 1: pathophysiology and risk factors. Nat Rev Cardiol.

28. Lanza GM, Yu X, Winter PM, Abendschein DR, Karukstis KK, Scott MJ, et al. Targeted antiproliferative drug delivery to vascular smooth muscle cells with a magnetic resonance imaging nanoparticle contrast agent implications for rational therapy of restenosis. Circulation. 2002;106(22):2842-7.

29. Winter PM, Neubauer AM, Caruthers SD, Harris TD, Robertson JD, Williams TA, et al. Endothelial alpha(v)beta3 integrin-targeted fumagillin nanoparticles inhibit angiogenesis in atherosclerosis. Arterioscler Thromb Vasc Biol. 2006;26(9):2103-9.

30. Winter PM, Caruthers SD, Zhang H, Williams TA, Wickline SA, Lanza GM. Antiangiogenic synergism of integrin-targeted fumagillin nanoparticles and atorvastatin in atherosclerosis. JACC Cardiovasc Imaging. 2008;1(5):624-34.
31. • Lobatto ME, Fayad ZA, Silvera S, Vucic E, Calcagno C, Mani V et al. Multimodal clinical imaging to longitudinally assess a nanomedical anti-inflammatory treatment in experimental atherosclerosis. Mol Pharm. 2010;7(6):2020-9. This study exemplifies that a theranostic approach can facilitate the development of nanotherapy.

32. McCarthy JR, Korngold E, Weissleder R, Jaffer FA. A lightactivated theranostic nanoagent for targeted macrophage ablation in inflammatory atherosclerosis. Small. 2010;6(18):2041-9.

33. Fitzgerald KT, Holladay CA, McCarthy C, Power KA, Pandit A, Gallagher WM. Standardization of models and methods used to assess nanoparticles in cardiovascular applications. Small. 2011;7 (6):705-17.

34. Bentzon JF, Falk E. Atherosclerotic lesions in mouse and man: is it the same disease? Curr Opin Lipidol. 2010;21(5):434-40.

35. Libby P, Ridker PM, Hansson GK. Progress and challenges in translating the biology of atherosclerosis. Nature. 2011;473 (7347):317-25.

36. Perazella MA. Current status of gadolinium toxicity in patients with kidney disease. Clin J Am Soc Nephrol. 2009;4(2):4619.

37. Chen J, Tung CH, Allport JR, Chen S, Weissleder R, Huang PL. Near-infrared fluorescent imaging of matrix metalloproteinase activity after myocardial infarction. Circulation. 2005;111 (14):1800-5.

38. Akinc A, Zumbuehl A, Goldberg M, Leshchiner ES, Busini V, Hossain N, et al. A combinatorial library of lipid-like materials for delivery of RNAi therapeutics. Nature Biotechnology. 2008;26 (5):561-9.

39. - Fayad ZA, Mani V, Woodward M, Kallend D, Abt M, Burgess T et al. Safety and efficacy of dalcetrapib on atherosclerotic disease using novel non-invasive multimodality imaging (dal-PLAQUE): a randomised clinical trial. Lancet. This is the first multicenter trial that uses noninvasive imaging to evaluate anti-atherosclerotic effects of a novel compound in patients. 